\title{
Leserforum
}

\section{NamensänderungEN der Gesellschaft - Dokumentation - DGD NfD DGI IWP}

\section{Offener Brief vom 3. Mai 2015 von Constantin Cazan}

DOI 10.1515/iwp-2015-0042

Sehr geehrter Vorstand, sehr geehrte Mitglieder,

der hier folgende Text entstand kurz nach Bekanntwerden der Absicht den Namen der Gesellschaft de novo zu ändern und richtet sich eig. an den früheren Präsidenten, Herrn Gradmann, der diese Namensänderung wesentlich kommuniziert hat, aber natürlich notabene auch an all jene, die das unterstützt oder befürwortet haben; insofern können sich all diese direkt angesprochen fühlen.

Eine breitere Diskussion zu der Namensänderung habe ich persönlich nicht wahrgenommen - vielleicht fand Sie in irgendwelchen Kreisen statt, die aber soweit ich das recherchiert habe - keine Spuren hinterließ.

In wenigen Tagen findet wieder eine Generalversammlung der Gesellschaft statt und ich fühle mich als Mitglied der Gesellschaft noch immer stark motiviert zu dokumentieren, dass ich mit dieser Namensänderung ebenso wie mit der früheren - nicht einverstanden bin. Auch wenn mir bewusst ist, dass das nichts ändern wird, möchte ich wenigstens diesbezüglich ein Zeichen setzen.

Am 15. Mai 1991 schrieb mir Arnoud de Kemp - wohl einen damaligen Standardbrief für neu eintretende Mitglieder - immerhin persönlich unterschrieben mit folgendem Wortlaut (Zitat):

\footnotetext{
„Die Gesellschaft für Dokumentation (DGD) e. V. versteht sich als Zuhause für alle, die sich beruflich oder während des Studiums mit Information und Dokumentation beschäftigen. Die DGD publiziert die Zeitschrift „Nachrichten für Dokumentation (NfD) und eine Reihe von Büchern, organisiert Tagungen und bietet ihnen die Möglichkeit in vielen Gremien und Arbeitskreisen Erfahrungen auszutauschen.“
}

Es ist einige Zeit her, dass das Editorial von Herrn Gradmann (IWP 4/2013) erschien, in dem die Umbenennung der Gesellschaft argumentiert wurde und auf das Luzian Weisel auf DGI News am 7.8.2013 hingewiesen hat mit Bitte um Kommentare. Obwohl ich keine explizite Diskussion dort wahrgenommen habe, fühle ich mich weiterhin stark motiviert diesem Editorial zu widersprechen.
Die Gesellschaft benennt sich also wieder einmal um. Das erscheint so wie Herr Gradmann und Herr Weins das betrachten und argumentieren zu stimmen - aber nur aus einer sehr speziellen höchstens Teil-Perspektive. Im Kern der Sache, der die Idee und den Zweck unserer Gesellschaft betrifft irren beide.

Im Sommer 2013 kam ich auf dem Weg entlang des Wiener Museumsquartiers an zwei Litfass (= Informations)säulen vorbei auf denen unter dem Titel 5uper.net „Artistic Bokeh research Wissensvermittlung und Forschung in neuer Medienkunst" und einem Photo mit den Teilen einer komplett zerlegten Digitalkamera folgender Text zu lesen war:

„Das Format „Dokumentation“ ist ein essentieller Part zur Reflexion, Präsentation und Strukturierung von Wissen ...

(vgl. http://5uper.net/post/193; letzter Zugriff am 15.3.2014)

Das gab mir zu Denken.

Während unsere Gesellschaft sich mehr und mehr vom Kern unserer Expertise und der Dokumentation entfernen und nach 1998 wieder über einen neuen Namen für die Gesellschaft nachdachte, wird der Begriff Dokumentation von Jüngeren neu entdeckt, definiert und belebt.

Das ist insofern spannend, weil in den letzten Jahrzehnten Dokumentation als „Begriff und terminus technicus“ in den medial gesteuerten Diskursen immer mehr verschwand „aus der Mode kam“, obwohl das von den verwendeten Informationssystemen und Berufswelten her, fachlich und methodisch überhaupt nicht gerechtfertigt ist. Nur als kleines Indiz sei hier erwähnt, dass der Begriff Dokumentation in der deutschen Wikipedia pro Jahr an die 50.000 Mal aufgerufen wird.

Ich habe nie verstanden, was die Gesellschaft von der Bezeichnung Dokumentation wegbrachte und dazu bewog, diese aus dem Vereinsnamen zu streichen. Wer den Markt unserer Gesellschaft kennt und sich die Mühe macht das detailliert zu beobachten, wird feststellen, dass es nach wie vor unzählige Institutionen und unzählige Menschen gibt, die Dokumentation (sic!) betreiben (siehe dazu auch die angeführten Beispiele - ja dass sich die 
Welt von der Dokumentation entferne, überhaupt nicht den Fakten entspricht und sich mit Wissen - als Handlungsexpertise! - gar nicht sinnhaft beschreiben lässt.

In fast jeder Ausgabe der Computerzeitschrift c't finden sich auf der Seite „Websites aktuell“ regelmäßig beeindruckende Beispiele für Dokumentation z. B. Bundeskreisliga.de, mueckenatlas.de, mosquitocatalog.org usw.

Hier sitzen Tausende Leute, die eine handelnde Expertise - Dokumentieren - pflegen. Hier wird nicht gewisst?!

Im Gegenteil ist festzuhalten, dass es zu „guter und gut gemachter Dokumentation ganz im Fugmannschen Sinne (vgl. iwp 58 (2007) 8,449-458 und 59(2008)3, 159164) bis heute keine relevante Alternative gibt (sic!).

Wissen ist ein seit Jahren inflationär verwendeter Begriff ohne Alleinstellungspofil, um die in der Gesellschaft versammelten Mitglieder markant und deutlich unterscheidbar von anderen Berufsgruppen hervorzuheben und $\mathrm{zu}$ charakterisieren. Wissen und mit Wissen agieren betrifft jede menschliche Gruppe.

Zugegeben wer sich der ständig tosenden Neuigkeitsbrandung des Webs und der forschenden Informationswissenschaft und Informatik rund um das Semantic Web, WebScience und der Ontologien aussetzt - Bereiche, deren Output - ob schriftlich oder gesprochen - häufig durch schlampige Terminologie und dokumentarisch schwach aufbereitete Publikationen (ohne Register und Indexe) auffallen - kann die Bedeutung klassischer Dokumentation aus dem Blickfeld verlieren.

Das sollte aber nicht dazu verleiten, weitgehend experimentelle Unternehmungen und Ideen zur Grundlage einer Entscheidung zu machen, die das Alleinstellungsmerkmal der DGD/DGI als einer praxisorientierten Berufs-Gesellschaft aufgibt zugunsten zeitgeistiger Modeerscheinungen und Begriffe.

Selbst Medline/PubMed, vielleicht demnächst die erste große Datenbank, die der semantischen Idee zu praxistauglicher Anwendung verhelfen könnte, ist nach 20 Jahren Arbeit daran noch immer nicht fertig. Wo immer man detailliert hinschaut zu funktionierenden Praxissystemen und Datenbanken sind weiterhin klassische dokumentarische Methoden im Einsatz (ZBMath, Embase, CAS usw.)

Auch 2012 - am Ende Ihrer Präsidentschaft, Herr Gradmann, gab es auf der Website der DGI keine durchgehende gemeinsame gut indexierte und aufbereitete Datenbank aller (!) NfD/IWP Beiträge, der Konferenzen, der Tagungen usw. mit einer effizienten Suchoberfläche, die alle Stückerln dokumentarischen Know-hows spiegelt und demonstriert.
Das bedeutet nicht, dass neue Entwicklungen keine Relevanz besitzen, aber der Weg neuer innovativer Ideen und Systeme zur alltäglichen Praxistauglichkeit ist weit und beschwerlich und den haben viele heute diskutierte Innovationen in der Informationsvermittlung noch vor sich - unter der zusätzlichen Hypothek einer medialen Innovationshysterie, die vielen Ideen gar nicht die Zeit gibt zu reifen, sondern gleich wieder von der nächsten unausgegorenen Entwicklung in Betaversion verdrängt wird.

Schließlich beinhalten die Umbenennungen noch zwei kontraproduktive Aspekte.

Der erste Aspekt betrifft die Identität der Gesellschaft: Wenn die Führung in einer ständigen Identitätskrise steckt, bekommen natürlich auch die Mitglieder früher oder später ein Problem. Gerade in den vergangenen Jahrzehnten hat unsere Profession mit der Bedrängung durch die Informatik zu tun, ohne dass irgendeine stabile und selbstbewusste Kontinuität und Gegenwehr unserer Community stattgefunden hat. Da braucht man eine Gesellschaft, die das Selbstbewusstsein und die öffentliche Wahrnehmung der Mitglieder in ihrer Expertise stärkt und sich nicht ständig wie eine Fahne im medialen Wind dreht.

Der zweite Aspekt betrifft klassische Betriebswirtschaft: Marketing und Branding.

DGD und NfD waren starke Marken, die weitläufig bekannt und eingängig waren; danach schon als Marken geschwächt DGI und IWP, die als solche erst wieder nach und nach neu aufgebaut „gebrandet“ werden mussten, um als breit bekannte Synonyme für unsere Tätigkeiten und Branche zu gelten, aber ohne Nettozuwachs an Bekanntheit gegenüber DGD/NfD-Zeiten, wie mir scheint. Diese Marken, und dazu zählen auch die Langformen der Namen, rührt man am besten gar nicht an, wenn man sich im Gedächtnis der Welt und der eigenen Märkte fest verankern will.

Wenn die Befürworterinnen und Befürworter der Namensänderungen mit ihren Argumenten Recht hätten, dann müssten Firmen wie IBM, Microsoft oder SAP oder unsere Schwesterorganisation die TEKOM und viele andere Unternehmen ständig ihren Namen wechseln. Das tun Sie aber nicht.

Der Name ist wie ein Logo, das man am besten unangetastet lässt und dessen einziger wesentlicher Zweck konstante(!) Wiedererkennung ist.

Die Entfernung und namentliche Distanzierung der Gesellschaft vom Begriff Dokumentation empfinde ich als Irrweg und Fehlentscheidung.

Auch wenn die Trefferangaben in Google nur Hochrechnungen sind und keine explizite Aussagekraft ha- 
ben, lässt sich leicht feststellen, dass es wenigstens ein paar Tausend Leute allein im deutschen Sprachraum geben sollte, die sich mit diesem Thema expertenhaft auseinandersetzen, sei es beruflich, sei es aus Interesse oder im Kontext betriebener Sammlungstätigkeit materieller oder ideeller Natur.

Die neuerlichen Änderungen im Namen der Gesellschaft und die offizielle Kommunikation vermitteln, dass wichtige Kernthemen - bedauerlicherweise - nicht (mehr) in dem Ausmaß vertreten und gepflegt werden, wie man es als an Dokumentation stark interessiertes Mitglied erwartet.

Mit freundlichen Grüßen

Constantin Cazan

Wien, Österreich

\section{Beispiele aktiv betriebener Dokumentationen}

\section{Österreich}

http://wirtschaftsgeschichte.univie.ac.at/vereine/doku/

Dokumentation lebensgeschichtlicher Aufzeichnungen http://www.wisdom.at/

http://bidok.uibk.ac.at/ behinderung inklusion dokumentation Die Dokumentation besteht seit 1930 und wertet die wichtigsten Wiener Zeitungen aus.

http://www.onb.ac.at/bibliothek/ariadne.htm Ariadne frauenspezifische Information und Dokumentation

Das Dokumentationsarchiv des Katholischen Jugendwerkes Österreichs gibt viermal im Jahr die Zeitschrift „Dokumentation“ heraus

http://www.vwgh.gv.at/rechtsprechung/dokeuroparecht.html Dokumentation für Europarecht http://www.tram.at/index.php/strassenbahngeschichte/ wtm-dokumentation

http://www.vhs.at/vhsarchiv-dokumentation.html

Theologische Literaturdokumentationen Innsbruck:

http://www.uibk.ac.at/theol/theoldi/index.html.de

http://bfw.dabis.org/PSI/init.psi Die BFW-Literaturdaten-

bank bietet eine umfassende Literaturdokumentation

über Forstwirtschaft in Österreich dem deutschspra-

chigen Raum.

http://www.uibk.ac.at/iza/ Innsbrucker Zeitungsarchiv

http://www.doew.at/erforschen/projekte/

datenbankprojekte Dokumentation des Österrei-

chischen Widerstands

\section{Deutschland}

http://www.berlin.de/lstu/dokumentation/thesaurus. html http://fpdf.de/dokumentation/

http://pubpsych.zpid.de/pubpsych/about.jsp

http://www.zbmath.org/software/

http://www.dkf-ev.de/ Dokumentation Kraftfahrwesen

http://www.miz.org/institutionen/forschung-

dokumentation

http://www.kathpedia.de/index.php?title= Nachkonziliare_Dokumentation

http://www.bach-digital.de/content/documentation.xml?

XSL.lastPage.SESSION=/content/documentation.xml http://www.caritasbibliothek.de/39328.html

Zeitschriftendokumentation Sozialwesen

http://geschichte.charite.de/aerztinnen/

http://www.baza-nazwisk.de/index.php

\section{Frankreich}

http://www.adbs.fr/centres-de-doc/

http://cdi.agro-bordeaux.fr/ 